\title{
Decompositions of the plane and the size of the continuum
}

\author{
by
}

Ramiro de la Vega (Bogotá)

\begin{abstract}
We consider a triple $\left\langle E_{0}, E_{1}, E_{2}\right\rangle$ of equivalence relations on $\mathbb{R}^{2}$ and investigate the possibility of decomposing the plane into three sets $\mathbb{R}^{2}=S_{0} \cup S_{1} \cup S_{2}$ in such a way that each $S_{i}$ intersects each $E_{i}$-class in finitely many points. Many results in the literature, starting with a famous theorem of Sierpiński, show that for certain triples the existence of such a decomposition is equivalent to the continuum hypothesis. We give a characterization in ZFC of the triples for which the decomposition exists. As an application we show that the plane can be covered by three sprays regardless of the size of the continuum, thus answering a question of J. H. Schmerl.
\end{abstract}

1. Introduction. In 1919, Sierpiński [7] proved that the continuum hypothesis $(\mathrm{CH})$ is equivalent to the existence of a subset $S$ of the plane such that each horizontal line intersects $S$ in countably many points and each vertical line intersects $S$ in co-countably many points. Later in [8] he proved that $\mathrm{CH}$ is equivalent to the statement that the three-dimensional euclidean space can be decomposed into three sets $S_{i}(i \in 3)$ in such a way that each line parallel to the $x_{i}$ axis intersects $S_{i}$ in finitely many points. After that, many mathematicians (see for example $[1-6,9,10]$ ) have found generalizations of these theorems in different directions. In [2] Erdős asked the following question which he attributed to Sierpiński: does there exist a set of three directions $d_{i}(i=1,2,3)$ in the plane, together with a decomposition of the plane into three corresponding sets $S_{i}$, such that every line in direction $d_{i}$ intersects $S_{i}$ in a finite set? A few years later Davies [1] showed that this is equivalent to $\mathrm{CH}$ and that it is irrelevant which directions you choose as long as they are different. More recently Komjáth [4] proved a similar equivalence where instead of three directions and lines in those directions one has three points and lines that pass through those points. Finally, in [6], Schmerl asks if the same equivalence holds when we consider

2000 Mathematics Subject Classification: Primary 03E50; Secondary 03E05, 51M05. Key words and phrases: continuum hypothesis, Sierpiński's theorem, sprays. 
three points and circles centered at those points. He remarks that $\mathrm{CH}$ does imply that there is such a decomposition but the question remains whether $\mathrm{CH}$ is indeed necessary in this case.

We find Schmerl's question very interesting because, as far as we know, all results related to Sierpiński's theorem deal essentially with linear objects in some euclidean space. However, the combinatorics of the problem is pretty much the same (whether it concerns lines or circles), at least at first sight. To state some context let us fix three equivalence relations $E_{i}, i \in 3$, on the plane with the property that for any $x \in \mathbb{R}^{2}$ and for $i, j \in 3$ with $i \neq j$ the set $[x]_{i} \cap[y]_{j}$ is finite (where $[x]_{i}$ denotes the equivalence class of $x$ $\bmod E_{i}$ ). We shall say that a decomposition of the plane $\mathbb{R}^{2}=\bigcup_{i \in 3} F_{i}$ is $\left\langle E_{0}, E_{1}, E_{2}\right\rangle$-acceptable if $[x]_{i} \cap F_{i}$ is finite for every $x \in \mathbb{R}^{2}$ and every $i \in 3$. In Davies' theorem, $E_{i}$ is the equivalence relation whose classes are precisely the lines with direction $d_{i}$, and he proves that the existence of an acceptable decomposition is equivalent to $\mathrm{CH}$. In Komjáth's theorem the equivalence classes $\bmod E_{i}$ are the lines that pass through a fixed point $p_{i}$, and again he proves that $\mathrm{CH}$ is equivalent to the existence of an acceptable decomposition of the plane. Finally, in Schmerl's question each $E_{i}$ is the relation "being at the same distance from $p_{i}$ " for some fixed centers $p_{i}(i \in 3)$. Intuitively, one should expect the same kind of theorem, but surprisingly only one of the implications is (trivially) true, namely that $\mathrm{CH}$ implies the existence of an acceptable decomposition.

Our main goal in this note is to characterize the triples for which there is an acceptable decomposition of the plane. Then we will use that characterization to explain the role of $\mathrm{CH}$ in the theorems of Davies, Komjáth and Sierpiński. Finally, in the last section we show how one can use that characterization to get in ZFC an acceptable decomposition of the plane for a specific triple, thus answering Schmerl's question.

2. Notation and terminology. We think of $\mathfrak{c}=|\mathbb{R}|$ as an initial ordinal. Let $T=\bigcup_{n \in \omega} \mathfrak{c}^{n}$ be the set of finite sequences of ordinals in $\mathfrak{c}$. We have two natural orders on $T$, the (partial) tree order $\subseteq$ and the lexicographic order $\leq$. In both orders we have the same minimum element $\Lambda$, the empty sequence. Given any $\sigma \in T$ and $\alpha \in \mathfrak{c}$ we write $\sigma^{\frown} \alpha=\sigma \cup\{\langle|\sigma|, \alpha\rangle\}$. For $\sigma \in T \backslash\{\Lambda\}$ we write $\sigma+1$ for the successor of $\sigma$ in the lexicographic order of $\mathfrak{c}^{|\sigma|}$; that is,

$$
\sigma+1=\left(\sigma\lceil(|\sigma|-1))^{\frown}(\sigma(|\sigma|-1)+1) .\right.
$$

We shall write $\sigma \wedge \tau$ for the infimum in the tree order of $\sigma$ and $\tau$; thus for $\sigma \neq \tau$ we have

$$
\sigma \wedge \tau=\sigma|| \sigma \wedge \tau|=\tau \Uparrow| \sigma \wedge \tau \mid \quad \text { and } \quad \sigma(|\sigma \wedge \tau|) \neq \tau(|\sigma \wedge \tau|) .
$$


Whenever we say that $M$ is an elementary submodel of the universe (and write $M \prec V$ ) we really mean that $(M, \in)$ is an elementary submodel of $(H(\theta), \in)$, where $H(\theta)$ is the set of all sets of hereditary cardinality less than $\theta$ and $\theta=\left(2^{\mathfrak{c}}\right)^{+}$. All the objects that we will consider (essentially points in $\mathbb{R}^{2}$, subsets of $\mathbb{R}^{2}$ and the collection of all subsets of $\mathbb{R}^{2}$ ) are in $H(\theta)$ and all the statements $\phi$ for which we shall use elementarity will be bounded so that in fact $M=\phi$ if and only if $\phi$ is true in the universe.

As mentioned in the introduction, we will consider triples $\left\langle E_{0}, E_{1}, E_{2}\right\rangle$ of equivalence relations on $\mathbb{R}^{2}$. For $x \in \mathbb{R}^{2}$, the equivalence class of $x$ $\bmod E_{i}$ will be denoted by $[x]_{i}$. All triples will have the property that $[x]_{i} \cap[x]_{j}$ is finite whenever $i \neq j$. We say that a decomposition $\mathbb{R}^{2}=$ $\bigcup_{i \in 3} F_{i}$ is $\left\langle E_{0}, E_{1}, E_{2}\right\rangle$-acceptable (or that the decomposition is acceptable for $\left.\left\langle E_{0}, E_{1}, E_{2}\right\rangle\right)$ if for every $x \in \mathbb{R}^{2}$ and $i \in 3$, the set $[x]_{i} \cap F_{i}$ is finite.

3. Twisted triples. We wish to characterize the triples $\left\langle E_{0}, E_{1}, E_{2}\right\rangle$ for which there exists an acceptable decomposition of the plane. Our main notion is the following:

Definition 3.1. We say that the triple $\left\langle E_{0}, E_{1}, E_{2}\right\rangle$ is twisted if for every $a \in \mathbb{R}^{2}$, for all $M, N \prec V$ such that $\left\langle E_{0}, E_{1}, E_{2}\right\rangle \in M \cap N$ and $N \in M$ and whenever $\{i, j, k\}=3$, we have

$$
\left|\left\{x \in[a]_{k}:[x]_{i} \in(M \backslash N),[x]_{j} \in(N \backslash M)\right\}\right|<\aleph_{0} .
$$

Our goal is to show that twisted triples are exactly the ones that admit acceptable decompositions. But first we need a coherent way to cover the plane with countable elementary submodels of the universe.

We fix $M_{\Lambda} \prec V$ such that $\mathbb{R}^{2} \cup\left\{E_{0}, E_{1}, E_{2}\right\} \subseteq M_{\Lambda}$ and $\left|M_{\Lambda}\right|=\mathfrak{c}$. Now we can find inductively (on the length of $\sigma \in T$ ) models $M_{\sigma} \prec V$ such that:

(i) the sequence $\left\langle M_{\sigma^{\wedge} \alpha}: \alpha \in \operatorname{cof}\left(\left|M_{\sigma}\right|\right)\right\rangle$ is a continuous (increasing) elementary chain,

(ii) $M_{\sigma} \subseteq \bigcup\left\{M_{\sigma^{\wedge} \alpha}: \alpha \in \operatorname{cof}\left(\left|M_{\sigma}\right|\right)\right\}$,

(iii) $\left\{E_{0}, E_{1}, E_{2}\right\} \cup\left\{M_{\tau}: \tau+1 \subseteq \sigma\right\} \subseteq M_{\sigma \sim 0}$,

(iv) $\left|M_{\tau}\right|>\left|M_{\sigma}\right|$ whenever $\tau \subset \sigma$ and $M_{\tau}$ is uncountable.

We actually do not need to define $M_{\sigma^{\wedge} \alpha}$ if $M_{\sigma}$ is countable or if $\alpha \geq$ $\operatorname{cof}\left(\left|M_{\sigma}\right|\right)$. On the other hand, note that conditions (ii) and (iv) imply that $\operatorname{rk}(x)$ is well defined as follows:

Definition 3.2. For $x \in M_{\Lambda}$ we $\operatorname{define} \operatorname{rk}(x)$ (the rank of $x$ ) as the minimum $\sigma \in T$ (in the lexicographic order) such that $M_{\sigma}$ is countable and $x \in M_{\tau}$ for all $\tau \subseteq \sigma$.

It is easy to see (using the continuity of the chains) that $\mathrm{rk}(x)$ is always a finite sequence of ordinals which are either successor ordinals or 0. Moreover, 
if $\sigma_{x}=\operatorname{rk}(x), \sigma_{y}=\operatorname{rk}(y), \sigma_{x}<\sigma_{y}$ and $n=\left|\sigma_{x} \wedge \sigma_{y}\right|$, then $\sigma_{y}(n)$ is a successor ordinal, say $\alpha+1$, and we define

$$
\Delta(x, y)=\left(\sigma_{x} \wedge \sigma_{y}\right)^{\frown} \alpha .
$$

The reason for this definition is that $M_{\Delta(x, y)}$ is in some sense the first model that witnesses the fact that $\operatorname{rk}(x)$ and $\operatorname{rk}(y)$ are different. Note that $\Delta(x, y)+1 \subseteq \operatorname{rk}(y)$ and that conditions (i) and (iii) imply that $M_{\Delta(x, y)} \in M_{\sigma}$ whenever $\sigma$ is a proper extension of $\Delta(x, y)+1$.

Observation 3.3. Given $x \in \mathbb{R}^{2}$ and $\sigma \in T$ note that if $x \in M_{\sigma}$ then by elementarity $\left\{[x]_{0},[x]_{1},[x]_{2}\right\} \subseteq M_{\sigma}$, so that $\operatorname{rk}\left([x]_{i}\right) \leq \operatorname{rk}(x)$ for all $i \in 3$; on the other hand, if $x \notin M_{\sigma}$ then by elementarity $\left|\left\{[x]_{0},[x]_{1},[x]_{2}\right\} \cap M_{\sigma}\right| \leq 1$ since $[x]_{i} \cap[x]_{j}$ is finite for $i \neq j$.

This simple fact will help us prove the following:

Lemma 3.4. If $\left\langle E_{0}, E_{1}, E_{2}\right\rangle$ is twisted then for each $x \in \mathbb{R}^{2}$ there is an $i \in 3$ such that $\operatorname{rk}\left([x]_{i}\right)=\operatorname{rk}(x)$.

Proof. First note that if $i \neq j$ and $\operatorname{rk}\left([x]_{i}\right)=\operatorname{rk}\left([x]_{j}\right)$ then necessarily $\operatorname{rk}(x)=\operatorname{rk}\left([x]_{i}\right)$, so we may assume without loss of generality that

$$
\operatorname{rk}\left([x]_{0}\right)<\operatorname{rk}\left([x]_{1}\right)<\operatorname{rk}\left([x]_{2}\right) ;
$$

we shall prove that in that case $\operatorname{rk}(x)=\operatorname{rk}\left([x]_{2}\right)$.

By Observation 3.3 we easily see that $\Delta\left([x]_{0},[x]_{2}\right)=\Delta\left([x]_{0}, x\right)$ and $\Delta\left([x]_{1},[x]_{2}\right)=\Delta\left([x]_{1}, x\right)$, and from these we get

$$
\begin{aligned}
& \Delta\left([x]_{0},[x]_{2}\right)+1 \subseteq \operatorname{rk}\left([x]_{2}\right) \wedge \operatorname{rk}(x), \\
& \Delta\left([x]_{1},[x]_{2}\right)+1 \subseteq \operatorname{rk}\left([x]_{2}\right) \wedge \operatorname{rk}(x) .
\end{aligned}
$$

Again by Observation 3.3 we have $\Delta\left([x]_{0},[x]_{2}\right)+1 \subset \Delta\left([x]_{1},[x]_{2}\right)$ and hence $M_{\Delta\left([x]_{0},[x]_{2}\right)} \in M_{\Delta\left([x]_{1},[x]_{2}\right)}$. We also have $[x]_{0} \in M_{\Delta\left([x]_{0},[x]_{2}\right)} \backslash M_{\Delta\left([x]_{1},[x]_{2}\right)}$ and $[x]_{1} \in M_{\Delta\left([x]_{1},[x]_{2}\right)} \backslash M_{\Delta\left([x]_{0},[x]_{2}\right)}$. But this means that, if $\sigma$ is a proper extension of $\operatorname{rk}\left([x]_{2}\right) \wedge \operatorname{rk}(x)$, then $M_{\Delta\left([x]_{0},[x]_{2}\right)}, M_{\Delta\left([x]_{1},[x]_{2}\right)} \in M_{\sigma}$, and therefore $x \in M_{\sigma}$ if and only if $[x]_{2} \in M_{\sigma}$. Here we have used the fact that $\left\langle E_{0}, E_{1}, E_{2}\right\rangle$ is twisted and therefore $x$ belongs to a finite set definable in terms of $[x]_{2}$, $M_{\Delta\left([x]_{0},[x]_{2}\right)}$ and $M_{\Delta\left([x]_{1},[x]_{2}\right)}$. This shows that $\operatorname{rk}(x)=\operatorname{rk}\left([x]_{2}\right)$.

LEMmA 3.5. If $\left\langle E_{0}, E_{1}, E_{2}\right\rangle$ is twisted then for every $a \in \mathbb{R}^{2}$, whenever $\{i, j, k\}=3$, the set

$$
X=\left\{x \in[a]_{k}: \operatorname{rk}\left([x]_{i}\right)<\operatorname{rk}\left([x]_{j}\right)<\operatorname{rk}\left([x]_{k}\right)=\operatorname{rk}(x)\right\}
$$

is finite.

Proof. As we noticed in the proof of the previous lemma, $x \in X$ implies that $[x]_{i} \in M_{\Delta\left([x]_{i},[x]_{k}\right)} \backslash M_{\Delta\left([x]_{j},[x]_{k}\right)},[x]_{j} \in M_{\Delta\left([x]_{j},[x]_{k}\right)} \backslash M_{\Delta\left([x]_{i},[x]_{k}\right)}$ and 
$M_{\Delta\left([x]_{i},[x]_{k}\right)} \in M_{\Delta\left([x]_{j},[x]_{k}\right)}$. Thus it is clear that

$$
X \subseteq \bigcup_{\substack{\sigma+1 \subseteq \operatorname{rk}\left([a]_{k}\right) \\ \tau+1 \subseteq \operatorname{rk}\left([a]_{k}\right)}}\left\{x \in[a]_{k}:[x]_{i} \in\left(M_{\sigma} \backslash M_{\tau}\right),[x]_{j} \in\left(M_{\tau} \backslash M_{\sigma}\right)\right\},
$$

and since $\left\langle E_{0}, E_{1}, E_{2}\right\rangle$ is twisted, the latter is a finite union of finite sets.

Now we need a way to order in type $\omega$ all the elements of $M_{\Lambda}$ of the same rank. This is easily done by fixing an injective enumeration

$$
M_{\sigma}=\left\{t_{n}^{\sigma}: n \in \omega\right\}
$$

for each $\sigma$ for which $M_{\sigma}$ is countable, and defining:

Definition 3.6. For $x \in M_{\Lambda}$ we define $\operatorname{deg}(x)$ (the degree of $x$ ) as the unique $n \in \omega$ for which $x=t_{n}^{\mathrm{rk}(x)}$.

The following is true for any triple $\left\langle E_{0}, E_{1}, E_{2}\right\rangle$, twisted or not.

Lemma 3.7. For every $a \in \mathbb{R}^{2}$ and for all $i, k \in 3$ with $i \neq k$, the set

$$
X=\left\{x \in[a]_{k}: \operatorname{rk}\left([x]_{i}\right)=\operatorname{rk}\left([x]_{k}\right) \text { and } \operatorname{deg}\left([x]_{i}\right)<\operatorname{deg}\left([x]_{k}\right)\right\}
$$

is finite.

Proof. Let $\sigma=\operatorname{rk}\left([a]_{k}\right)$ and $n=\operatorname{deg}\left([a]_{k}\right)$. Note that if $x \in X$ then there is an $m<n$ (namely, $m=\operatorname{deg}\left([x]_{i}\right)$ ) such that $x \in t_{m}^{\sigma} \cap t_{n}^{\sigma}$ and $t_{m}^{\sigma} \cap t_{n}^{\sigma}$ is finite. Hence $X$ is contained in a finite union of finite sets.

Finally, we are ready to prove the main result of this section.

THEOREM 3.8. The following are equivalent:

(1) The triple $\left\langle E_{0}, E_{1}, E_{2}\right\rangle$ is twisted.

(2) There is an $\left\langle E_{0}, E_{1}, E_{2}\right\rangle$-acceptable decomposition of $\mathbb{R}^{2}$.

Proof. Suppose first that the triple $\left\langle E_{0}, E_{1}, E_{2}\right\rangle$ is twisted and for each $k \in 3$ define $F_{k}$ as the set of all $x \in \mathbb{R}^{2}$ such that:

(i) $\operatorname{rk}(x)=\operatorname{rk}\left([x]_{k}\right)$,

(ii) for all $i \in 3 \backslash\{k\}$, if $\operatorname{rk}\left([x]_{i}\right)=\operatorname{rk}\left([x]_{k}\right)$ then $\operatorname{deg}\left([x]_{i}\right)<\operatorname{deg}\left([x]_{k}\right)$.

Given $x \in \mathbb{R}^{2}$ we know by Lemma 3.4 that $I:=\left\{i \in 3: \operatorname{rk}(x)=\operatorname{rk}\left([x]_{i}\right)\right\}$ in not empty. If we let $k \in I$ be such that $\operatorname{deg}\left([x]_{i}\right) \leq \operatorname{deg}\left([x]_{k}\right)$ for all $i \in I$, then it is immediate from the construction that $x \in F_{k}$. Hence $\mathbb{R}^{2}=$ $F_{0} \cup F_{1} \cup F_{2}$.

On the other hand, using Lemmas 3.5 and 3.7 we see that for any $a \in \mathbb{R}^{2}$ and $k \in 3$ the set $[a]_{k} \cap F_{k}$ is finite, so that the decomposition $\mathbb{R}^{2}=F_{0} \cup$ $F_{1} \cup F_{2}$ is indeed $\left\langle E_{0}, E_{1}, E_{2}\right\rangle$-acceptable.

Now suppose (2) holds and $\left\langle E_{0}, E_{1}, E_{2}\right\rangle$ is not twisted. This means that there are $M, N \prec V$ with $\left\langle E_{0}, E_{1}, E_{2}\right\rangle \in M \cap N$ and $N \in M$ such that for 
some $a \in \mathbb{R}^{2}$ and some $\{i, j, k\}=3$ the set

$$
X=\left\{x \in[a]_{k}:[x]_{i} \in(M \backslash N),[x]_{j} \in(N \backslash M)\right\}
$$

is infinite. On the other hand, using elementarity and the fact that $N \in M$, we can find $F_{0}, F_{1}, F_{2} \in M \cap N$ such that $\mathbb{R}^{2}=F_{0} \cup F_{1} \cup F_{2}$ is $\left\langle E_{0}, E_{1}, E_{2}\right\rangle$ acceptable. Now for each $x \in X$ we have $x \notin M$ because $[x]_{j} \notin M$ and therefore $x \notin F_{i}$, since otherwise $x$ would be in a finite set definable from $F_{i}$ and $[x]_{i}$ which are both in $M$. Similarly, $x \notin N$ and hence $x \notin F_{j}$. But then $X \subseteq F_{k}$, which contradicts the fact that $[a]_{k} \cap F_{k}$ is finite.

4. The role of $\mathrm{CH}$. In principle, the fact that a given triple is twisted (or untwisted) should depend only on the geometry of the triple. However, the presence of $\mathrm{CH}$ trivializes things as we now show.

Theorem 4.1. Under $C H$ every triple $\left\langle E_{0}, E_{1}, E_{2}\right\rangle$ is (trivially) twisted.

Proof. Let $a \in \mathbb{R}^{2}$, let $M, N \prec V$ be such that $\left\langle E_{0}, E_{1}, E_{2}\right\rangle \in M \cap N$ and $N \in M$, and let $\{i, j, k\}=3$. It is well known that either $N \cap \omega_{1}$ is countable or $\omega_{1} \subseteq N$. Under CH this implies that either $\mathbb{R}^{2} \cap N$ is countable or $\mathbb{R}^{2} \subseteq N$. If $\mathbb{R}^{2} \cap N$ is countable then since $N \in M$ we have $\mathbb{R}^{2} \cap N \subseteq M$, so there is no $x \in \mathbb{R}^{2}$ for which $[x]_{j} \in N \backslash M$. On the other hand, if $\mathbb{R}^{2} \subseteq N$ then there is no $x \in \mathbb{R}^{2}$ for which $[x]_{i} \in M \backslash N$. In either case the set

$$
\left\{x \in[a]_{k}:[x]_{i} \in M \backslash N,[x]_{j} \in N \backslash M\right\}
$$

is empty, and hence $\left\langle E_{0}, E_{1}, E_{2}\right\rangle$ is twisted.

As an immediate consequence of Theorems 3.8 and 4.1 we get the following result which is already known (for instance, it is a special case of Theorem 2 in [3], for $\theta=1$ and $(r, s)=(2,1))$ :

Corollary 4.2. Under $C H$ every triple $\left\langle E_{0}, E_{1}, E_{2}\right\rangle$ admits an acceptable decomposition.

Under $\neg \mathrm{CH}$ the situation is quite different. Say we want to show that a certain triple is not twisted. Now we can just take $M, N \prec V$ with $|M|=\aleph_{0}$ and $\left|\mathbb{R}^{2} \cap N\right|=\aleph_{1}<\mathfrak{c}$ and it is usually the case that there is $x \in \mathbb{R}^{2}$ for which, say, $[x]_{0} \in M \backslash N$ and $[x]_{1} \in N \backslash M$. Of course, there may still be cases where this does not happen (e.g. if all the classes are finite) and again the triple will be twisted for trivial reasons. But for the triples we have mentioned (the ones associated with Davies', Komjáth's and Sierpiński's theorems and the triple associated with Schmerl's question) we are in that situation.

The question now is whether you can "move" the point $x$ in such a way that $[x]_{2}$ remains constant while $[x]_{0}$ and $[x]_{1}$ change in a "definable way" (not depending on $[x]_{2}$ ) so that they do not get out of $M$ and $N$ respectively. In Sierpiński's theorem (here we are working in $\mathbb{R}^{3}$ instead of $\mathbb{R}^{2}$ but that 
does not make any difference) this task is trivial because here $[x]_{i}$ is just the line that passes through $x$ and is parallel to the $e_{i}$ axis, so we can just add any rational number to the third coordinate of $x$ and we are done. Similarly, in Davies's theorem, $[x]_{i}$ is the line in direction $d_{i}$ that passes through $x$; now we just need to move $x$ in direction $d_{2}$ by a rational amount. The task is not as immediate in Komjáth's theorem and it requires a rather clever trigonometric argument.

We illustrate in more detail these ideas with the following result which is half of the answer to Schmerl's question. According to Schmerl [6], a subset $S \subseteq \mathbb{R}^{2}$ is a spray around $c \in \mathbb{R}^{2}$ if the intersection of $S$ with any circle centered at $c$ is finite.

TheOREM 4.3. Let $c_{i}$ for $i \in 3$ be three distinct points on $\mathbb{R}^{2}$ that lie on the same line. Then the following are equivalent:

(1) $\mathrm{CH}$.

(2) $\mathbb{R}^{2}$ can be covered by three sprays around the points $c_{i}(i \in 3)$.

Proof. We define $E_{i}$ as the set of all $(x, y) \in \mathbb{R}^{2} \times \mathbb{R}^{2}$ such that $\left\|x-c_{i}\right\|=$ $\left\|y-c_{i}\right\|$. Now (1) implies (2) by Corollary 4.2. Assume then that $\mathfrak{c}>\aleph_{1}$. We are done if we can prove that $\left\langle E_{0}, E_{1}, E_{2}\right\rangle$ is not twisted.

For $i \in 2$ fix rational intervals $I_{i} \subseteq \mathbb{R}$ such that whenever $C_{i}$ is a circle centered at $c_{i}$ with radius $r_{i} \in I_{i}$ then $C_{0} \cap C_{1} \neq \emptyset$.

Now let $M, N \prec V$ with $|M|=\aleph_{0}$ and $|\mathbb{R} \cap N|=\aleph_{1}<\mathfrak{c}$ be such that $\left\{c_{0}, c_{1}, c_{2}\right\} \subseteq M \cap N$ and $N \in M$. Since $\mathbb{R} \backslash N \neq \emptyset$ and $N \in M$ we deduce by elementarity that there is an $r_{0} \in I_{0} \cap(M \backslash N)$. On the other hand, $\left|I_{1} \cap N\right|=\aleph_{1}>|M|$ so there is an $r_{1} \in I_{1} \cap(N \backslash M)$. Let $x \in \mathbb{R}^{2}$ be such that $\left\|x-c_{i}\right\|=r_{i}$ for $i \in 2$. Then

$$
(t-1)\left\|x-c_{2}\right\|^{2}=t r_{0}^{2}-r_{1}^{2}+t\left(\left\|c_{2}\right\|^{2}-\left\|c_{0}\right\|^{2}\right)+\left\|c_{1}\right\|^{2}-\left\|c_{2}\right\|^{2}
$$

where $t$ is the unique real number for which $c_{1}-c_{2}=t\left(c_{0}-c_{2}\right)$. What is important is that $t \in M \cap N$, so that for every large enough $n \in \omega$ we have $r_{0, n}:=\sqrt{r_{0}^{2}+1 / n} \in I_{0} \cap(M \backslash N), r_{1, n}:=\sqrt{r_{1}^{2}+t / n} \in I_{1} \cap(N \backslash M)$ and any point $x_{n} \in \mathbb{R}^{2}$ for which $\left\|x_{n}-c_{i}\right\|=r_{i, n}$ for $i \in 2$ (which exists by the definition of $I_{0}$ and $I_{1}$ ) will satisfy $\left\|x_{n}-c_{2}\right\|=\left\|x-c_{2}\right\|$. Therefore $\left\langle E_{0}, E_{1}, E_{2}\right\rangle$ is not twisted.

5. The plane can be covered by three sprays. The goal of this section is to show (in ZFC) that if $c_{0}, c_{1}, c_{2} \in \mathbb{R}^{2}$ are the vertices of an equilateral triangle, then the plane can be covered by three sprays $S_{0}, S_{1}$ and $S_{2}$ around $c_{0}, c_{1}$ and $c_{2}$ respectively. We suspect that the same remains true for any triangle (as long as the $c_{i}$ 's do not lie on the same line) and we have checked a couple of examples, but we have not found a reasonable way to 
prove it simultaneously because in one step of the proof we require a computer algebra system to check for the irreducibility of a certain polynomial.

We may assume without loss of generality (applying first a circle preserving transformation if necessary) that $c_{0}=(-1,0), c_{1}=(1,0)$ and $c_{2}=(0, \sqrt{3})$. Suppose that three circles $C_{0}, C_{1}$ and $C_{2}$ centered at $c_{0}$, $c_{1}$ and $c_{2}$ respectively have a point in common. If we use $\alpha_{0}, \alpha_{1}$ and $\alpha_{2}$ for the squares of their radii we obtain the relation

$$
\alpha_{0}^{2}+\alpha_{1}^{2}+\alpha_{2}^{2}-\alpha_{0} \alpha_{1}-\alpha_{0} \alpha_{2}-\alpha_{1} \alpha_{2}-4 \alpha_{0}-4 \alpha_{1}-4 \alpha_{2}+16=0 .
$$

Now suppose that $C_{0}^{\prime}$ and $C_{1}^{\prime}$ are also circles centered at $c_{0}$ and $c_{1}$ respectively, have radii $\beta_{0}$ and $\beta_{1}$, and $C_{0}^{\prime}, C_{1}^{\prime}$ and $C_{2}$ also have a point in common. Then we have a similar equation (involving $\beta_{0}, \beta_{1}$ and $\alpha_{2}$ ) and we can eliminate $\alpha_{2}$ to get a relation of the form

$$
p\left(\alpha_{0}, \beta_{0}, \alpha_{1}, \beta_{1}\right)=0
$$

where $p=p(X, Y, Z, W)$ is a polynomial of degree 4 . We want to think of $X, Y$ as variables and regard $Z, W$ as parameters. It is not worth it to write down here what $p$ exactly is; instead we state without proof the facts about $p$ that we will be needing. First of all one can use a computer algebra system (e.g. MAPLE) to check that $p(X, Y, 1,2)$ is absolutely irreducible as a polynomial in two variables. On the other hand, one can prove that for any such polynomial, the set of $(z, w) \in \mathbb{R}^{2}$ for which $p(X, Y, z, w)$ is not absolutely irreducible is either the whole $\mathbb{R}^{2}$ or is contained in a curve. Since we already know that the former is not the case, we obtain the following:

Lemma 5.1. If $A \subseteq \mathbb{R}$ is infinite then there exist $z, w \in A$ such that $p(X, Y, z, w)$ is absolutely irreducible as a polynomial in $X, Y$.

Now suppose that we have two pairs of parameters $(z, w)$ and $\left(z^{\prime}, w^{\prime}\right)$ such that both $p(X, Y, z, w)$ and $p\left(X, Y, z^{\prime}, w^{\prime}\right)$ are irreducible. If $(z, w) \neq$ $\left(z^{\prime}, w^{\prime}\right)$ we can check by simple inspection that these two polynomials are not constant multiples of each other. This implies the following:

Lemma 5.2. Suppose that $z, z^{\prime}, w, w^{\prime} \in \mathbb{R},(z, w) \neq\left(z^{\prime}, w^{\prime}\right)$ and that both $p(X, Y, z, w)$ and $p\left(X, Y, z^{\prime}, w^{\prime}\right)$ are absolutely irreducible. Then the system

$$
\begin{aligned}
p(X, Y, z, w) & =0, \\
p\left(X, Y, z^{\prime}, w^{\prime}\right) & =0
\end{aligned}
$$

has finitely many solutions.

We are ready to prove that $\left\langle E_{0}, E_{1}, E_{2}\right\rangle$ is twisted, where for each $i \in 3$, $E_{i}$ is the equivalence relation in $\mathbb{R}^{2}$ whose classes are circles centered at $c_{i}$. If $x \in \mathbb{R}^{2}$ and $i \in 3$ we define $r_{i}(x):=\left\|x-c_{i}\right\|^{2}$. Fix $M, N \prec V$ with $\left\langle E_{0}, E_{1}, E_{2}\right\rangle \in M \cap N$ and $N \in M$. Since the triangle $c_{0} c_{1} c_{2}$ is equilateral, 
it is enough to prove that

$$
B(a):=\left\{x \in[a]_{2}:[x]_{0} \in(M \backslash N),[x]_{1} \in(N \backslash M)\right\}
$$

is finite for any $a \in \mathbb{R}^{2}$. Suppose for contradiction that there is $a \in \mathbb{R}^{2}$ for which $B(a)$ is infinite. Then $A:=\left\{r_{1}(x): x \in B(a)\right\}$ is an infinite subset of $\mathbb{R}$ and therefore by Lemma 5.1 there are $\alpha_{1}, \beta_{1} \in A$ such that $p\left(X, Y, \alpha_{1}, \beta_{1}\right)$ is absolutely irreducible. By the definition of $A$ there are $x, x^{\prime} \in[a]_{2}$ such that $\alpha_{1}=r_{1}(x)$ and $\beta_{1}=r_{1}\left(x^{\prime}\right)$, and we let $\alpha_{0}=r_{0}(x)$ and $\beta_{0}=r_{0}\left(x^{\prime}\right)$. Now let $G$ be the set of all $(\alpha, \beta) \in \mathbb{R} \times \mathbb{R}$ such that:

(i) $\alpha, \beta \in N$,

(ii) there are $z, z^{\prime} \in \mathbb{R}^{2}$ such that $r_{0}(z)=\alpha_{0}, r_{0}\left(z^{\prime}\right)=\beta_{0}, r_{1}(z)=\alpha$, $r_{1}\left(z^{\prime}\right)=\beta$ and $r_{2}(z)=r_{2}\left(z^{\prime}\right)$

(iii) the polynomial $p(X, Y, \alpha, \beta)$ is absolutely irreducible.

Note that $G \in M$ since it is definable in terms of $N, \alpha_{0}$ and $\beta_{0}$ which are all in $M$. Also note that $\left(\alpha_{1}, \beta_{1}\right) \in G$ so that $G \neq \emptyset$. By elementarity there is $\left(\alpha_{1}^{\prime}, \beta_{1}^{\prime}\right) \in G \cap M$ and since $\left(\alpha_{1}, \beta_{1}\right) \notin M$ we have $\left(\alpha_{1}, \beta_{1}\right) \neq\left(\alpha_{1}^{\prime}, \beta_{1}^{\prime}\right)$. Finally, by Lemma 5.2 we deduce that the system

$$
\begin{aligned}
& p\left(X, Y, \alpha_{1}, \beta_{1}\right)=0, \\
& p\left(X, Y, \alpha_{1}^{\prime}, \beta_{1}^{\prime}\right)=0
\end{aligned}
$$

has finitely many solutions. But this system is definable in $N$ and therefore $\left(\alpha_{0}, \beta_{0}\right)$, being one of its solutions, must belong to $N$, a contradiction. Hence $\left\langle E_{0}, E_{1}, E_{2}\right\rangle$ is twisted.

Using Theorem 3.8 we have just proved the following (no assumptions on the size of the continuum):

THEOREM 5.3. If $c_{0}, c_{1}, c_{2} \in \mathbb{R}^{2}$ are the vertices of an equilateral triangle, then the plane can be covered by three sprays $S_{0}, S_{1}$ and $S_{2}$ around $c_{0}, c_{1}$ and $c_{2}$ respectively.

\section{References}

[1] R. Davies, On a problem of Erdös concerning decompositions of the plane, Proc. Cambridge Philos. Soc. 59 (1963), 33-36.

[2] P. Erdős, Some remarks on set theory IV, Michigan Math. J. 2 (1953-54), 169-173.

[3] P. Erdős, S. Jackson and R. D. Mauldin, On partitions of lines and space, Fund. Math. 145 (1994), 101-119.

[4] P. Komjáth, Three clouds may cover the plane, Ann. Pure Appl. Logic 109 (2001), $71-75$.

[5] C. Kuratowski, Sur une caractérisation des alephs, Fund. Math. 38 (1951), 14-17.

[6] J. H. Schmerl, How many clouds cover the plane?, ibid. 177 (2003), 209-211.

[7] W. Sierpiński, Sur un théorème équivalent à l'hypothèse du continu $\left(2^{\aleph_{0}}=\aleph_{1}\right)$, Bull. Int. Acad. Polon. Sci. Lett. Cl. Sci. Math. Nat. Sér. A Sci. Math. 1919, 1-3. 
[8] W. Sierpiński, Sur une propriété paradoxale de l'espace à trois dimensions équivalente à l'hypothèse du continu, Rend. Circ. Mat. Palermo (2) 1 (1952), 7-10.

[9] R. Sikorski, A characterization of alephs, Fund. Math. 38 (1951), 18-22.

[10] J. C. Simms, Sierpiński's theorem, Simon Stevin 65 (1991), 69-163.

Universidad de los Andes

Bogotá, Colombia

E-mail: rade@uniandes.edu.co

Received 1 October 2008;

in revised form 10 December 2008 